\title{
Proliferative vitreoretinopathy: revised concepts of pathogenesis and adjunctive treatment
}

\author{
David G. Charteris ${ }^{1}$
}

Received: 27 September 2019 / Accepted: 27 September 2019 / Published online: 27 November 2019

(c) The Royal College of Ophthalmologists 2019

\begin{abstract}
Proliferative vitreoretinopathy (PVR) remains a significant challenge for vitreoretinal surgeons. Its incidence, as a complication of retinal detachment, does not appear to have altered despite improvement in surgical techniques. Extensive laboratory research has been undertaken to investigate the pathogenesis of PVR and the use of adjunctive mediations to modify the disease process. To date these studies have not resulted in improved clinical management of PVR. Previous concepts of the pathogenesis of PVR, focussing on a central role for RPE cells, may be flawed and could potentially have contributed to the lack of clinical advances. Revised clinical classifications and novel approaches to adjunctive treatment may in the future result in improved surgical outcomes.
\end{abstract}

\section{Introduction}

Proliferative vitreoretinopathy (PVR) remains the most common cause of final failure after retinal detachment repair. The term PVR was introduced as part of a unifying definition and staging by the Retina Society Terminology Committee in 1983 [1]. An incidence of 5-10\% of all rhegmatogenous retinal detachments was given in this publication. Despite advances in surgical techniques and the understanding of vitreoretinal pathology there is no evidence that the incidence of PVR as a complication of retinal detachment repair has changed in the intervening years [2-4].

The visual results of successful repair of retinal detachment with PVR were described in a detailed report by Andenmatten and Gonvers [5]. This demonstrated that surgical repair was time consuming (an average of 3.8 procedures taking $5 \mathrm{~h}$ of operating time) and the results frequently unsatisfactory-patients were unlikely to regain stereopsis and often had visual comfort described as

This study was presented at the Cambridge Eye Symposium "Fibrosis and the Eye" 5-6 September 2019.

David G. Charteris

d.charteris@nhs.net

1 The Institute of Ophthalmology, Moorfields Eye Hospital, City Road, London EC1V 2PD, UK "medium to bad". The authors concluded that "successful vitreoretinal surgery seems to have been of little or no benefit" to most of their patients. At this time Lewis et al. had described moderately successful primary anatomical success rates for PVR surgery ( $81 \%$ for initial surgery and $68 \%$ for repeat surgery) but noted limited visual recovery in these series [6, 7]. The Silicone Oil Study reported limited primary success rates for PVR surgery (35-42\%) and again poor visual recovery [8].

The introduction of modern vitreoretinal surgery enabled more detailed investigations into the pathogenesis of PVR on tissues removed at the time of surgery. A concept of contractile peri-retinal membranes centred on proliferating RPE cells and containing smaller populations of glial, (nonRPE) fibroblastic and inflammatory cells was developed [9]. This was the foundation for the identification of possible targets for adjunctive agents to improve both the anatomical and visual outcome of PVR surgery.

\section{Adjunctive treatment of PVR}

The proliferative nature of the PVR process led to an interest in the anti-proliferative agent 5 fluorouracil (5 FU) as an adjunct to PVR surgery. Blumenkranz demonstrated that $5 \mathrm{FU}$ reduced tractional retinal detachment in a PVR animal model [10] and that $5 \mathrm{FU}$ was well tolerated in the posterior segment as part of vitreoretinal surgery [11]. Advances in the understanding of the pathology of PVR and 
the need identified by surgical results subsequently resulted in numerous laboratory and pre-clinical studies in the search for potential adjunctive treatments [9, 12, 13]. Very few laboratory studies on potential adjuncts for PVR have advanced to clinical assessment.

\section{Clinical trials on PVR adjuncts}

Randomised controlled trials (RCT) have been undertaken on only a small number of the adjuncts tested in pre-clinical studies. The effect of post-operative irradiation was tested in a small-scale randomised trial using $300 \mathrm{cGy}$ in divided doses [14]. No beneficial effect of the irradiation was seen although the patient numbers were limited. Similarly, a small scale RCT on a combination of heparin and dexamethasone in vitrectomy infusion fluid failed to show a significant effect in established PVR although the patients numbers may not have adequate to test the treatment [15].

The efficacy of the anti-proliferative agent daunomycin in established PVR has been investigated in a large scale multi-centre RCT [16]. PVR patients were randomised to treatment with or without a $10 \mathrm{~min}$ intraoperative infusion of daunomycin during vitrectomy. Although the primary outcome measure failed to reach significance the trial did find a statistically significant reduction in the number of vitreoretinal re-operations within 1 year-for the first time demonstrating an adjunctive treatment effect on the PVR process. Despite this potentially advantageous clinical outcome daunomycin has not found widespread clinical use possibly because of concerns over safety, difficulty in obtaining and utilising an anti-proliferative agent or due to a lack of beneficial effects when tested in other units.

In the UK a series of studies were undertaken to assess the potential of a combination $5 \mathrm{FU}$ and low-molecular weight heparin (LMWH), given as a per-operative infusion, to influence the development of PVR. $5 \mathrm{FU}$ is known to be anti-proliferative against RPE cells and has been shown to reduce experimental tractional retinal detachment [9]. LMWH reduces post-vitrectomy fibrin, inhibits RPE proliferation, binds fibrogenic growth factors and reduces experimental tractional retinal detachment [9]. An initial RCT on high-risk retinal detachments demonstrated a significant reduction in post-operative PVR [17] suggesting that this adjunctive combination could play an important role in vitreoretinal surgery. Further studies on this combination did not, however, show a beneficial effect on established PVR [18] or on unselected primary retinal detachments [4]. This adjunctive combination has also failed to gain widespread clinical acceptance-probably for similar reasons to those outlined above for daunomycin.

\section{Adjunctive steroids}

Analysis of human PVR retinae has demonstrated a marked upregulation of retinal glia intermediate filament staining suggesting a central role for the glial response to injury in PVR development (Fig. 1a) [13, 19, 20]. Experimentally it has been noted that after retinal detachment the Muller glia extension into the subretinal space is consistently related to glial proliferation [21]. We investigated the potential of the steroid triamcinolone acetonide (TA) to modify the retinal response to injury.

Briefly, to assess the effect of TA on experimental retinal detachment localised detachments were induced in pigmented adult rabbits [22]. Triamcinolone $(2 \mathrm{mg})$ was given intravitreally (a) at the time of detachment and (b) $24 \mathrm{~h}$ later. To allow assessment of proliferation BrdU was injected $4 \mathrm{~h}$ prior to sacrifice at 3 days after detachment induction. Retinas were examined using a panel of probes to detect cellular activation of Muller cells and microglia (antivimentin and isolectin B4, respectively) and proliferation (anti-BrdU). Total cell counts were analysed for proliferating cells (BrdU positive cells/mm). Vehicle injected 3 day retinal detachments without triamcinolone were studied as controls. Intra-retinal glial activation and photoreceptor deconstruction was seen in both treated and control animals. BrdU localised to retinal glial cells in control animals. Triamcinolone treatment resulted in a significant reduction in BrdU positive cell counts from a mean of 21 cells $/ \mathrm{mm}$ retina to 7 cells $/ \mathrm{mm}$ retina. Proliferating Muller cell (cell nuclei in INL) were reduced from a mean of $19 / \mathrm{mm}$ retina to 4 . There was no evidence of Muller cell or microglial activation in non-detached retina. Occasional RPE cells were BrdU positive (Fig. 1b, c).

It was notable that although triamcinolone reduced glial cell proliferation in acute retinal detachment, Muller cell hypertrophy was not affected suggesting that this is a separate event from proliferation. We concluded that adjunctive steroid treatment had the potential to modify the acute retinal response to detachment and thus could influence the development of PVR. Subsequently we have undertaken clinical studies on the use of adjunctive steroids in PVR.

\section{Clinical studies on steroids in PVR}

Previous small scale, uncontrolled clinical studies of PVR have suggested that systemic prednisolone [23], infused dexamethasone [15] and intravitreal triamcinolone [24-26] may reduce the severity of PVR although none of these studies was of sufficient power to provide a definitive answer.

Sustained delivery of an adjunctive treatment has the advantage of maintaining an active concentration of drug 
Fig. 1 a Confocal microscopy of human PVR retinectomy specimen. Glial intermediate filament stain (GFAP) green and rhodopsin stain (red). Note marked upregulation of glial intermediate filament expression throughout retina. The photoreceptor inner and outer segments have been lost and glial cell processes have extended through the outer limiting membrane (white arrow) to form a confluent subretinal glial scar (yellow arrow). Rhodopsin has been redistributed to rod cell bodies and rods have extended neurite processes towards the inner retina (blue arrows). b, c Experimental retinal detachment (3 day, rabbit). Anti-Vimentin (green) labels Muller cells. There is an increase in labelling within Muller cells after detachment. Anti-BrdU (red) labels dividing cells. Isolectin B4 (blue) labels microglia. b (Control, untreated) proliferating glial (Muller) cells are demonstrated by the red stain (anti BrdU) in the inner nuclear layer. There is an increase in dividing Muller cells (in the INL) and microglia (one shown in the OS layer). c Treated with $2 \mathrm{mg}$ triamcinolone on detachment induction and at $24 \mathrm{~h}$ note the marked reduction in glial cell proliferation (from a mean of 21 cells $/ \mathrm{mm}$ retina to 7 cells/mm retina)

\section{a}

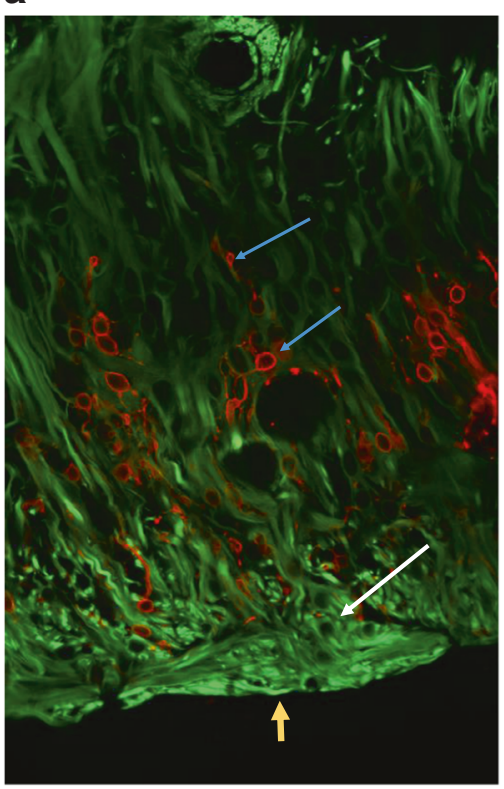

b

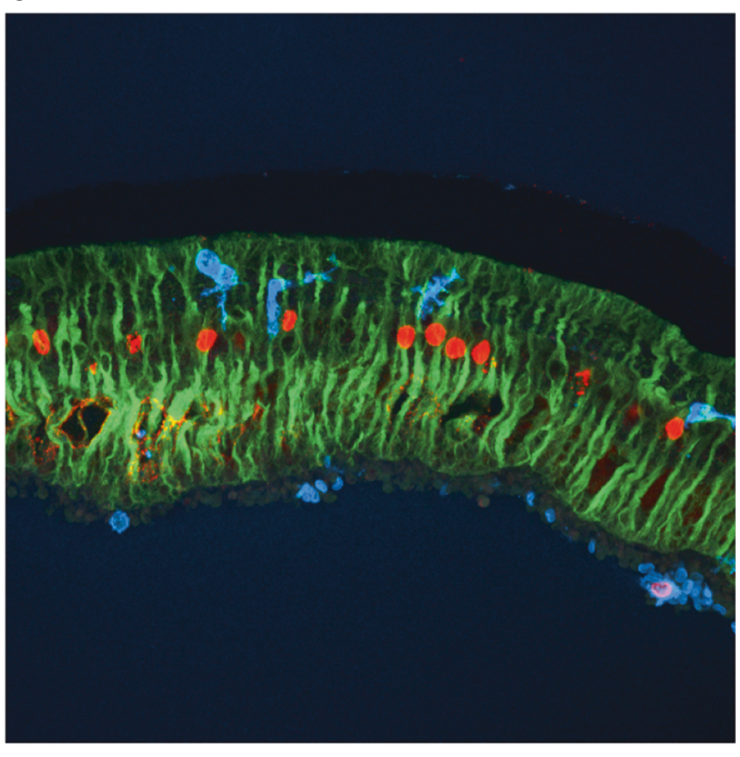

C

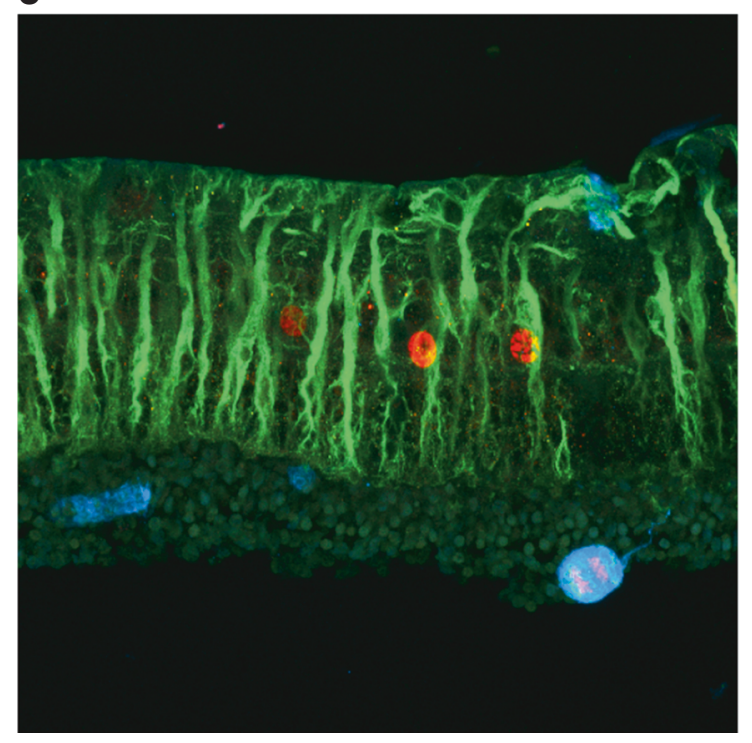

within the vitreous cavity throughout the active phase of PVR development [9]. We used a $0.7 \mathrm{mg}$ slow-release preparation of dexamethasone (Ozurdex, Allergan) in an RCT of eyes with established (grade C) PVR at the time of initial surgery and of oil removal to establish whether this preparation can improve outcomes of vitreoretinal surgery for grade C PVR. Anatomical outcomes were not improved however there was a significant reduction in cystoid macular oedema in the treated group [27].

PVR is common, and often severe, following penetrating ocular trauma and we have investigated the potential of TA to improve outcomes in a small-scale randomised study of eyes undergoing vitrectomy for open-globe trauma [28]. Although anatomical outcomes were similar in treatment and control groups, visual acuity was better in the TA group

suggesting role for TA as an adjunct in open-globe trauma. Subsequently we have initiated a large scale multi-centre study on the use of TA in penetrating trauma cases undergoing vitrectomy surgery (the ASCOT study) [29]. This investigation will run over a 6-year period, report in 2020 and recruit 300 cases from centres around the UK.

\section{Reasons for the lack of progress in PVR treatments}

Despite 40 years of laboratory and clinical investigations there is an obvious absence of new approaches to PVR management. Currently there are no widely accepted adjunctive treatments and PVR remains a surgical disease. 
Reviewing the potential reasons for this is therefore a priority. Broadly these fall in to two areas: (a) a lack of understanding the basic pathobiology of the condition and related to this flawed animal models, and (b) inadequate clinical classification and standardised surgical strategies.

Models of the pathogenesis of PVR have historically focused on a central role for migrating RPE cells $[9,12,13]$. Whilst RPE cells are consistently present in PVR periretinal membranes their role may have been overestimated. Initial studies on human PVR analysed membranes obtained by surgical peeling from PVR eyes-these studies reinforced the central role of RPE cells. Examining PVR retinectomies, where there is a larger sample of membrane tissue and its underlying relationship to the retina can be studied, results in a different view of PVR pathogenesis $[19,20]$. Glial cells dominate the peri-retinal tissues and their continuation to the intra-retinal glia is notable. Hence the previous analyses of surgically peeled membrane tissue may have only looked at an inner lamina of the membrane (populated by free-floating RPE cells), underestimating the glial role due to a form of sampling artefact. This has clear implications for the design of adjunctive treatments but also implies that many of the previously utilised, RPE cell generated, animal models are of little value.

The clinical classifications of PVR most commonly used are the Retina Society Terminology Committee classification of 1983 [1] and its revision in 1991 [30, 31]. These classifications have limitations-they are based entirely on anatomical descriptions at a single time point and do not take into account the biological activity of the PVR process. Grades A and B in these classifications describe early changes that form a continuum with uncomplicated retinal detachment, may be subtle and are open to subjective interpretation. Furthermore, they do not take into account possible relevant clinical features such as the duration of the process - it was notable that in our study on the Ozurdex implant $30 \%$ of cases (of established grade C PVR) had not had previous vitreoretinal surgery [27]. Ultimately the currently used classifications have not contributed to recent progress in PVR management and review and revision would appear appropriate. The genetic background of PVR cases has recently been investigated and has been suggested as one potential aspect of future PVR classification [13].

The analysis of surgical outcomes is another potential avenue to an improved clinical classification of PVR. We utilised data from our previous large scale study on adjuncts in unselected primary retinal detachment cases to estimate the risk of failure from PVR and provide a simple points system to grade the risk of PVR developing [32]. Risk factors and outcomes were analysed using a multiple variable logistic regression model. This identified three risk factors that contributed to the risk of failure by PVR: vitreous haemorrhage, grade C PVR on presentation and extent of detachment. These can be combined as part of a simplified points system and provide a test of relatively high diagnostic accuracy for failure due to PVR. Diagnostic accuracy was estimated by the area under the receiver operating characteristic (ROC) curve as 0.84 .

\section{Conclusions}

PVR continues to occur and remains a challenge for vitreoretinal surgeons. Adjunctive treatment aimed at improving anatomical and visual outcomes has been proposed since the early stages of modern vitreoretinal surgery, however, to date results have been disappointing. An inadequate understanding of the pathogenesis of PVR, with a focus on the RPE cell contribution, may have contributed to this. Future adjunctive strategies targeted at the retinal response may produce better outcomes. Notably a recent study using mitomycin $\mathrm{C}$, given at the time of vitrectomy, to directly target the retinal response in severe intraocular foreign body injuries appeared to reduce the incidence of PVR [33] and this approach deserves further investigation.

Other aspects of PVR research require modified approaches to produce new treatments. Clinical classifications may need revision and attention to sub-classification of PVR cases for clinical trials may be useful. More unified surgical approaches (and outcomes) may be needed for the adequate investigation of adjunctive treatments. Clinical trials are time consuming and resource intensive and advances in the clinical assessment and surgical management PVR would appear to be currently necessary to enable future clinical investigations.

Acknowledgements The research was funded by National Institute for Health Research Health Technology Assessment Programme, Moorfields Eye Hospital Special Trustees, Allergan Pharmaceuticals and Royal College of Surgeons of Edinburgh/Royal Blind and supported by the NIHR Biomedical Research Centre at Moorfields Eye Hospital NHS Foundation Trust and UCL Institute of Ophthalmology and the NIHR Moorfields Clinical Research Facility. The views expressed are those of the author(s) and not necessarily those of the NHS, the NIHR or the Department of Health. Dr Geoffrey P Lewis supplied the images

Funding The author has received grants for research on PVR from the following sources: National Institute for Health Research Health Technology Assessment Programme. Moorfields Eye Hospital Special Trustees. Allergan Pharmaceuticals. Royal College of Surgeons of Edinburgh/Royal Blind. NIHR Biomedical Research Centre at Moorfields Eye Hospital and UCL Institute of Ophthalmology.

\section{Compliance with ethical standards}

Conflict of interest The author declares no conflict of interest.

Publisher's note Springer Nature remains neutral with regard to jurisdictional claims in published maps and institutional affiliations. 


\section{References}

1. The Retina Society Terminology Committee. The classification of retinal detachment with proliferative vitreoretinopathy. Ophthalmology. 1983;90:121-5.

2. Joeres S, Kirchhof B, Joussen AM. PVR as a complication of rhegmatogeneous retinal detachment: a solved problem? Br J Ophthalmol. 2006;90:796-7.

3. Heimann H, Bartz-Schmidt KU, Bornfeld N, Weiss C, Hilgers $\mathrm{RD}$, Foerster MH, Scleral Buckling versus Primary Vitrectomy in Rhegmatogenous Retinal Detachment Study Group. Scleral buckling versus primary vitrectomy in rhegmatogenous retinal detachment: a prospective randomized multicenter clinical study. Ophthalmology. 2007;114:2142-54.

4. Wickham L, Bunce C, Wong D, McGurn D, Charteris DG. A randomised controlled trial of combined 5-fluorouracil and low molecular weight heparin in the management of unselected rhegmatogenous retinal detachments undergoing primary vitrectomy. Ophthalmology. 2007;114:698-704.

5. Andenmatten R, Gonvers M. Sophisticated vitreoretinal surgery in patients with a healthy fellow eye. An 11-year retrospective study. Graefes Arch Clin Exp Ophthalmol. 1993;231:495-9.

6. Lewis H, Aaberg TM, Abrams GW. Causes of failure after initial vitreoretinal surgery for severe proliferative vitreoretinopathy. Am J Ophthalmol. 1991;111:8-14.

7. Lewis $\mathrm{H}$, Aaberg TM. Causes of failure after repeat vitreoretinal surgery for recurrent proliferative vitreoretinopathy. Am J Ophthalmol. 1991;111:15-9.

8. Silicone Study Group. Vitrectomy with silicone oil or perfluoropropane gas in eyes with severe proliferative vitreoretinopathy: results of a randomized clinical trial. Silicone study report 2. Arch Ophthalmol. 1992;110:780-92.

9. Charteris DG. Proliferative vitreoretinopathy: pathobiology, surgical management and adjunctive treatment. $\mathrm{Br} \mathrm{J}$ Ophthalmol. 1995;79:953-60.

10. Blumenkranz MS, Ophir A, Claflin AJ, Hajek A. Fluorouracil for the treatment of massive periretinal proliferation. Am J Ophthalmol. 1982;94:458-67.

11. Blumenkranz M, Hernandez E, Ophir A, Norton EW. 5-fluorouracil: new applications in complicated retinal detachment for an established antimetabolite. Ophthalmology. 1984;91:122-30.

12. Charteris DG, Sethi CS, Lewis GP, Fisher SK. Proliferative vitreoretinopathy-developments in adjunctive treatment and retinal pathology. Eye. 2002;16:369-74.

13. Pastor JC, Rojas J, Pastor-Idoate S, Di Lauro S, Gonzalez-Buendia L, Delgado-Tirado S. Proliferative vitreoretinopathy: a new concept of disease pathogenesis and practical consequences. Prog Retin Eye Res. 2016;51:125-55.

14. Binder S, Bonnet M, Velikay M, Gerard JP, Stolba U, Wedrich A, et al. Radiation therapy in proliferative vitreoretinopathy. A prospective randomized study. Graefes Arch Clin Exp Ophthalmol. 1994;232:211-4.

15. Williams RG, Chang S, Comaratta MR, Simoni G. Does the presence of heparin and dexamethasone in the vitrectomy infusate reduce reproliferation in proliferative vitreoretinopathy? Graefes Arch Clin Exp Ophthalmol. 1996;234:496-503.

16. Wiedemann P, Hilgers RD, Bauer P, Heimann K. Adjunctive Daunorubicin in the treatment of proliferative vitreoretinopathy: results of a multicenter clinical trial. Am J Ophthalmol. 1998; 126:550-9.

17. Asaria RH, Kon CH, Bunce C, Charteris DG, Wong D, Khaw PT, et al. Adjuvant 5-fluorouracil and heparin prevents proliferative vitreoretinopathy: results from a randomized, double-blind, controlled clinical trial. Ophthalmology. 2001;108:1179-83.
18. Charteris DG, Aylward GW, Wong D, Groenewald C, Asaria RHY, Bunce C. A randomized controlled trial of combined 5fluorouracil and low-molecular-weight heparin in management of established proliferative vitreoretinopathy. Ophthalmology. 2004; 111:2240-5.

19. Sethi CS, Lewis GP, Fisher SK, Leitner WP, Mann DL, Luthert $\mathrm{PJ}$, et al. Glial remodelling and neural plasticity in human retinal detachment with proliferative vitreoretinopathy. Investig Ophthalmol Vis Sci. 2005;46:329-42.

20. Charteris DG, Downie J, Aylward GW, Sethi C, Luthert P. Intraretinal and periretinal pathology in anterior proliferative vitreoretinopathy. Graefes Arch Clin Exp Ophthalmol. 2007;245: 93-100.

21. Lewis GP, Chapin EA, Luna G, Linberg KA, Fisher SK. The fate of Müller's glia following experimental retinal detachment: nuclear migration, cell division, and subretinal glial scar formation. Mol Vis. 2010;16:1361-72.

22. Charteris DG, Lewis GP, Fisher SK. Triamcinolone reduces intraretinal proliferation in acute experimental retinal detachment. Investig Ophthalmol Vis Sci. 2008;49:4887.

23. Koerner F, Merz A, Gloor B, Wagner E. Postoperative retinal fibrosis-a controlled clinical study of systemic steroid therapy. Graefes Arch Clin Exp Ophthalmol. 1982;219:268-71.

24. Munir WM, Pulido JS, Sharma MC, Buerk BM. Intravitreal triamcinolone for treatment of complicated proliferative diabetic retinopathy and proliferative vitreoretinopathy. Can J Ophthalmol. 2005;40:598-604.

25. Cheema RA, Peyman GA, Fang T, Jones A, Lukaris AD, Lim K. Triamcinolone acetonide as an adjuvant in the surgical treatment of retinal detachment with proliferative vitreoretinopathy. Ophthalmic Surg Lasers Imaging. 2007;38:365-70.

26. Ahmadieh H, Feghhi M, Tabatabaei H, Shoeibi N, Ramezani A, Mohebbi MR. Triamcinolone acetonide in silicone-filled eyes as adjunctive treatment for proliferative vitreoretinopathy: a randomized clinical trial. Ophthalmology. 2008;115:1938-43.

27. Banerjee PJ, Quartilho A, Bunce C, Xing W, Zvobgo TM, Harris $\mathrm{N}$. et al. Slow-release dexamethasone in proliferative vitreoretinopathy: a prospective, randomized controlled clinical trial. Ophthalmology. 2017;124:757-67.

28. Banerjee PJ, Xing W, Bunce C, Woodcock M, Chandra A, Scott $\mathrm{RA}$, et al. Triamcinolone during pars plana vitrectomy for open globe trauma: a pilot randomised controlled clinical trial. Br J Ophthalmol. 2016;100:949-55.

29. Banerjee PJ, Cornelius VR, Phillips R, Lo JW, Bunce C, Kelly J, et al. Adjunctive intraocular and peri-ocular steroid (triamcinolone acetonide) versus standard treatment in eyes undergoing vitreoretinal surgery for open globe trauma (ASCOT): study protocol for a phase III, multi-centre, double-masked randomised controlled trial. Trials. 2016;17:339.

30. Machemer R, Aaberg TM, Freeman HM, Irvine AR, Lean JS, Michels RM. An updated classification of retinal detachment with proliferative vitreoretinopathy. Am J Ophthalmol. 1991;112: 159-65.

31. Di Lauro S, Kadhim MR, Charteris DG, Pastor JC. Classifications for proliferative vitreoretinopathy (PVR): an analysis of their use in publications over the last 15 years. J Ophthalmol. 2016;2016: 7807596

32. Wickham L, Bunce C, Wong D, Charteris DG. Retinal detachment repair by vitrectomy: simplified formulae to estimate the risk of failure. Br J Ophthalmol. 2011;95:1239-44.

33. Assi A, Khoueir Z, Helou C, Fakhoury H, Cherfan G. Intraocular application of Mitomycin $\mathrm{C}$ to prevent proliferative vitreoretinopathy in perforating and severe intraocular foreign body injuries. Eye. 2019;33:1261-70. 